\title{
Unifying Search-based and Compilation-based Approaches to Multi-agent Path Finding through Satisfiability Modulo Theories
}

\author{
Pavel Surynek \\ FIT, Czech Technical University in Prague, Thákurova 9, 16000 Praha 6, Czechia \\ pavel.surynek@ fit.cvut.cz
}

\begin{abstract}
We unify search-based and compilation-based approaches to multi-agent path finding (MAPF) through satisfiability modulo theories (SMT). The task in MAPF is to navigate agents in an undirected graph to given goal vertices so that they do not collide. We rephrase Conflict-Based Search (CBS), one of the state-of-the-art algorithms for optimal MAPF solving, in the terms of SMT. This idea combines SAT-based solving known from MDDSAT, a SAT-based optimal MAPF solver, at the low-level with conflict elimination of CBS at the high-level. Where the standard CBS branches the search after a conflict, we refine the propositional model with a disjunctive constraint. Our novel algorithm called SMT-CBS hence does not branch at the high-level but incrementally extends the propositional model. We experimentally compare SMTCBS with CBS, ICBS, and MDD-SAT.
\end{abstract}

\section{Introduction and Background}

Multi-agent path finding in graphs (MAPF) [Standley, 2010; Yu and LaValle, 2016] represents an important problem in artificial intelligence with specific applications in planning and robotics. We assume multiple distinguishable agents placed in vertices of an undirected graph such that at most one agent is placed in each vertex. Agents can be moved between vertices across edges. MAPF usually assumes that agents are moved to unoccupied neighbors. The task in MAPF is to reach a given goal configuration of agents from a given starting configuration using valid movements.

We address optimal solving of MAPF with respect to common cumulative objective functions that are minimized sum-of-costs [Sharon et al., 2013; Miltzow et al., 2016] and makespan [Yu and LaValle, 2016]. The sum-of-costs corresponds to the total cost of all movements (including wait actions) until the goal configuration in reached - traversal of an edge and wait actions have unit cost. The makespan calculates the total number of time-steps until the goal is reached.

Many practical problems from robotics can be interpreted as MAPF. Examples include discrete multi-robot navigation and coordination, item rearrangement in automated warehouses [Basile et al., 2012], ship collision avoidance [Kim et al., 2014], or formation maintenance and maneuvering of aerial vehicles [Zhou and Schwager, 2015].

This paper contributes by the design and experimental analysis of a novel optimal MAPF algorithm that unifies two major approaches to solving MAPF optimally: a searchbased approach represented by conflict-based search (CBS) [Sharon et al., 2015] and a compilation-based approach represented by reducing MAPF to propositional satisfiability (SAT) [Biere et al., 2009] in the MDD-SAT algorithm [Surynek et al., 2016]. Our novel algorithm called SMT-CBS rephrases ideas of CBS in the terms of satisfiability modulo theories (SMT) [Bofill et al., 2012] at the high-level. While at the low-level we use the SAT encoding from MDD-SAT.

Unlike the original CBS that resolves conflicts between agents by branching the search, SMT-CBS refines the propositional model with a disjunctive constraint to resolve the conflict. SMT-CBS hence does not branch at the high-level but instead incrementally extends the propositional model that is consulted with the external SAT solver similarly as it has been done in MDD-SAT. In contrast to MDD-SAT where the propositional model is fully constructed in a single-shot, the propositional model is being built lazily in SMT-CBS as new conflicts appear.

The hypothesis behind the design of SMT-CBS is that in many cases we do not need to add all constraints to form the complete propositional model while still be able to obtain a conflict-free solution. Intuitively we expect that such cases where the incomplete propositional model will suffice are represented by sparsely occupied instances with large environments. The expected benefit in contrast to MDD-SAT is that incomplete model can be constructed and solved faster. On the other hand we expect that the superior performance of MDD-SAT in environments densely populated with agents will be preserved as SMT-CBS will quickly converge the model towards the complete one.

We first introduce MAPF formally. Then CBS and MDDSAT are recalled. On top of this, the combination of CBS and MDD-SAT is developed - the SMT-CBS algorithm. Finally an experimental evaluation of SMT-CBS against CBS , ICBS, and MDD-SAT on various benchmarks is presented.

\subsection{MAPF Formally}

Multi-agent path finding (MAPF) [Silver, 2005; Ryan, 2008] consists of an undirected graph $G=(V, E)$ and a set of 

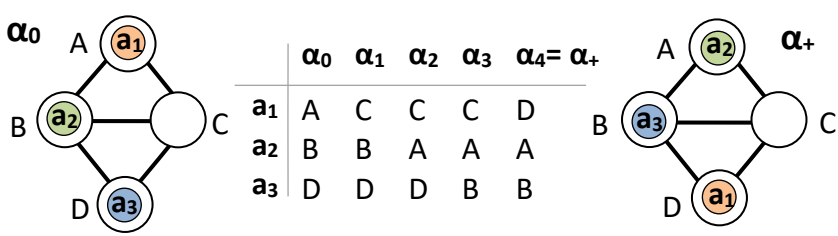

Figure 1: A MAPF instance with three agents $a_{1}, a_{2}$, and $a_{3}$.

agents $A=\left\{a_{1}, a_{2}, \ldots, a_{k}\right\}$ such that $|A|<|V|$. At most one agent resides in each vertex. The configuration of agents is denoted $\alpha: A \rightarrow V$. Starting configuration of agents $\alpha_{0}$ and goal configuration $\alpha_{+}$are specified too.

At each time step, an agent can either move to an adjacent vertex or wait in its current vertex. The task is to find a sequence of move/wait actions for each agent $a_{i}$ that moves the agent from $\alpha_{0}\left(a_{i}\right)$ to $\alpha_{+}\left(a_{i}\right)$ such that agents do not collide, i.e., do not occupy the same vertex at the same time. Typically, an agent can move into adjacent unoccupied vertex provided no other agent enters the same target vertex. However different rules for movements are used in variants of MAPF. An example of MAPF instance is shown in Figure 1.

We develop all new concepts in this paper for the move-tounoccupied variant formalized in the following definition.

Definition 1 (move-to-unoccupied MAPF). Configuration $\alpha^{\prime}$ is a valid successor of $\alpha$ if and only if the following conditions hold:

(i) $\alpha(a)=\alpha^{\prime}(a)$ or $\left\{\alpha(a), \alpha^{\prime}(a)\right\} \in E$ for all $a \in A$ (agents wait or move along edges);

(ii) for all $a \in A$ it holds that if $\alpha(a) \neq \alpha^{\prime}(a)$ then $\alpha^{\prime}(a) \neq \alpha\left(a^{\prime}\right)$ for all $a^{\prime} \in A$ (target vertex is empty);

(iii) and for all $a, a^{\prime} \in A$ it holds that if $a \neq a^{\prime}$ then $\alpha^{\prime}(a) \neq \alpha^{\prime}\left(a^{\prime}\right)$ (no two agents enter the same target).

Solving MAPF is to find a sequence of configurations $\left[\alpha_{0}, \alpha_{1}, \ldots, \alpha_{\mu}\right]$ such that $\alpha_{i+1}$ results using valid movements from $\alpha_{i}$ for $i=1,2, \ldots, \mu-1$, and $\alpha_{\mu}=\alpha_{+}$. A feasible solution of a solvable MAPF instance can be found in polynomial time [Wilson, 1974; Kornhauser et al., 1984]; precisely the worst case time complexity of most practical algorithms for finding feasible solutions is $\mathcal{O}\left(|V|^{3}\right)$ [Luna and Bekris, 2011; de Wilde et al., 2014].

\subsection{Cumulative Objectives in MAPF}

We are often interested in optimal solutions. In case of the makespan [Surynek, 2017] we just need to minimize $\mu$ in the aforementioned solution sequence. For introducing the sumof-costs objective [Dresner and Stone, 2008; Standley, 2010; Sharon et al., 2013] we need more notation as follows:

Definition 2 Sum-of-costs objective is the summation, over all $k$ agents, of the number of time steps required to reach the goal vertex. Denoted $\xi$, where $\xi=\sum_{i=1}^{k} \xi\left(\right.$ path $\left.\left(a_{i}\right)\right)$ and $\xi\left(\right.$ path $\left.\left(a_{i}\right)\right)$ is an individual path cost of agent $a_{i}$ connecting $\alpha_{0}\left(a_{i}\right)$ and $\alpha_{+}\left(a_{i}\right)$ calculated as the number of edge traversals and wait actions disregarding any final wait actions. ${ }^{1}$

\footnotetext{
${ }^{1}$ The notation path $\left(a_{i}\right)$ refers to path in the form of a sequence of vertices and edges connecting $\alpha_{0}\left(a_{i}\right)$ and $\alpha_{+}\left(a_{i}\right)$ while $\xi$ assigns the cost to a given path.
}

We note that finding a solution that is optimal (minimal) with respect to either the makespan or the sum-of-costs objective is NP-hard [Ratner and Warmuth, 1986; Surynek, 2010].

\section{Unifying Search and Compilation}

A necessary step before introducing the unification between the search-based and the compilation-based approach is to briefly discuss both approaches themselves.

\subsection{Conflict-based Search}

CBS is a representative of search-based approach. CBS uses the idea of resolving conflicts lazily; that is, a solution of MAPF instance is not searched against the complete set of movement constraints that forbids collisions between agents but with respect to initially empty set of collision forbidding constraints that gradually grows as new conflicts appear. The advantage of CBS is that it can find a valid solution before all constraints are added.

The high-level of CBS searches a constraint tree (CT) using a priority queue in breadth first manner. CT is a binary tree where each node $N$ contains a set of collision avoidance constraints $N$.constraints - a set of triples $\left(a_{i}, v, t\right)$ forbidding occurrence of agent $a_{i}$ in vertex $v$ at time step $t$, a solution $N$.paths - a set of $k$ paths for individual agents, and the total cost $N . \xi$ of the current solution.

The low-level process in CBS associated with node $N$ searches paths for individual agents with respect to set of constraints $N$.constraints. For a given agent $a_{i}$, this is a standard single source shortest path search from $\alpha_{0}\left(a_{i}\right)$ to $\alpha_{+}\left(a_{i}\right)$ that avoids a set of vertices $\left\{v \in V \mid\left(a_{i}, v, t\right) \in N\right.$. constraints $\}$ whenever working at time step $t$. For details see [Sharon $e t$ al., 2015].

CBS stores nodes of CT into priority queue OPEN sorted according to the ascending costs of solutions. At each step CBS takes node $N$ with the lowest cost from OPEN and checks if $N$.paths represent paths that are valid with respect to MAPF movements rules - that is, N.paths are checked for collisions. If there is no collision, the algorithms returns valid MAPF solution N.paths. Otherwise the search branches by creating a new pair of nodes in CT - successors of $N$. Assume that a collision occurred between agents $a_{i}$ and $a_{j}$ in vertex $v$ at time step $t$. This collision can be avoided if either agent $a_{i}$ or agent $a_{j}$ does not reside in $v$ at timestep $t$. These two options correspond to new successor nodes of $N-N_{1}$ and $N_{2}$ that inherit the set of conflicts from $N$ as follows: $N_{1}$. conflicts $=N$. conflicts $\cup\left\{\left(a_{i}, v, t\right)\right\}$ and $N_{2}$. conflicts $=N$. conflicts $\cup\left\{\left(a_{j}, v, t\right)\right\} . \quad N_{1}$. paths and $N_{1}$.paths inherit paths from N.paths except those for agents $a_{i}$ and $a_{j}$ respectively. Paths for $a_{i}$ and $a_{j}$ are recalculated with respect to extended sets of conflicts $N_{1}$. conflicts and $N_{2}$. conflicts respectively and new costs for both agents $N_{1} . \xi$ and $N_{2} . \xi$ are determined. After this, $N_{1}$ and $N_{2}$ are inserted into the priority queue OPEN.

The pseudo-code of CBS is listed as Algorithm 1. One of crucial steps occurs at line 16 where a new path for colliding agents $a_{i}$ and $a_{j}$ is constructed with respect to the extended set of conflicts. N.paths $(a)$ refers to path of agent $a$.

The CBS algorithm ensures finding sum-of-costs optimal solution (see detailed proofs in [Sharon et al., 2015]). 


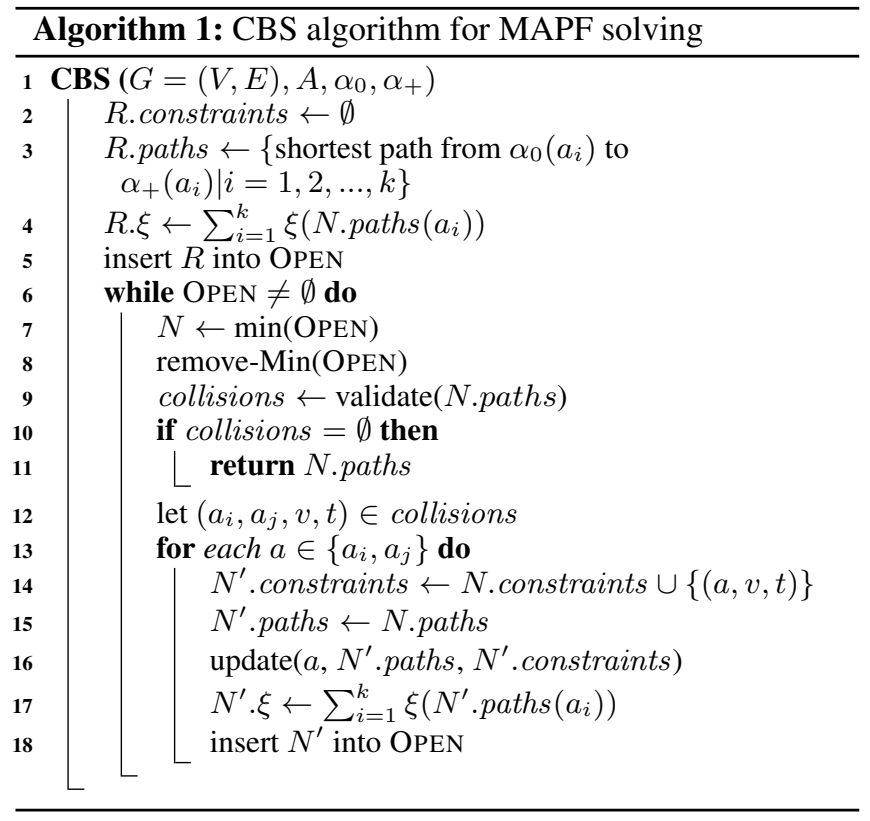

\section{Implications for SMT-CBS}

A deterministic implementation of the non-deterministic selection of collision at line 12 has a great impact on the performance. A considerable research effort has been devoted to select collisions with respect to their importance, to this end cardinal and semi-cardinal collisions have been defined [Boyarski et al., 2015; Felner et al., 2018].

There are two important observations about the CBS algorithm that we further hypothetise to be important in SMTCBS. Assume fixed time-step $t$ :

Observation 1 All potential conflicts considering all agents $a_{1}, a_{2}, \ldots, a_{k}$, and all vertices $v_{1}, v_{2}, \ldots, v_{n}$ that can appear within CBS together form an at-most-one occupation constraint: $\left|\left\{a_{i} \mid \alpha_{t}\left(a_{i}\right)=v_{j}, i=1,2, \ldots k\right\}\right| \leq 1$ for each $j=1,2, \ldots, n$.

In other words, the above at-most-one constraint, let us denote it as $O C C_{\leq 1}$, says there is at most one agent per vertex.

Observation 2 It may happen that a set of paths found by $C B S$ at node $N$ such that $N$.contraints $\subset O C C_{\leq 1}$ is consistent with respect to $O C C_{\leq 1}$.

The observation formalizes what we generally hope for when using CBS. It can discover a solution before all potential constraints are added which leads to faster solving.

\subsection{Compilation to Propositional Satisfiability}

The major alternative to CBS is represented by compilation of MAPF to propositional satisfiability (SAT) [Surynek et al., 2016; Surynek, 2017]. The idea follows SAT-based planning [Kautz and Selman, 1999] where the existence of a plan for a fixed number time steps is modeled as SAT. We similarly construct propositional formula $\mathcal{F}(\xi)$ such that it is satisfiable if and only if a solution of a given MAPF of sum-of-costs $\xi$ exists. Moreover, the approach is constructive; that is, $\mathcal{F}(\xi)$ exactly reflects the MAPF instance and if satisfiable, solution

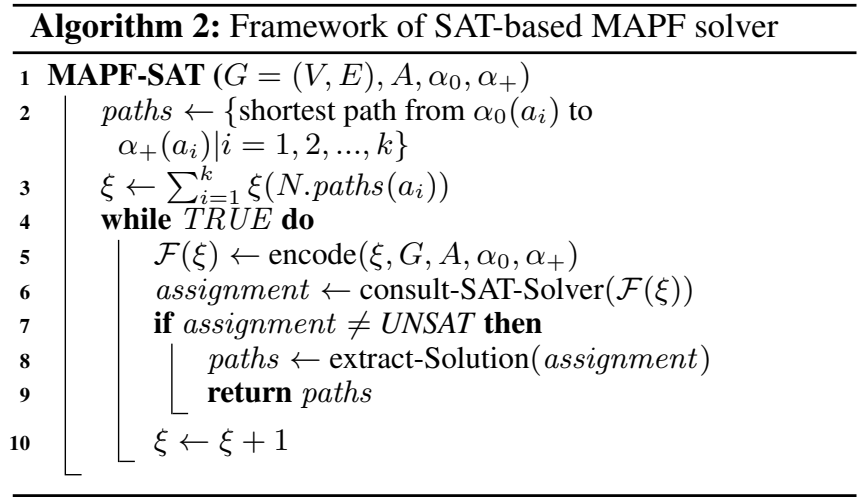

of MAPF can be reconstructed from satisfying assignment of the formula. We say $\mathcal{F}(\xi)$ to be a complete propositional model of MAPF.

Definition 3 (complete propositional model). Propositional formula $\mathcal{F}(\xi)$ is a complete propositional model of $M A P F \Sigma$ if the following condition holds:

\section{$\mathcal{F}(\xi)$ is satisfiable $\Leftrightarrow \Sigma$ has a solution of sum-of-costs $\xi$.}

Being able to construct such formula $\mathcal{F}$ one can obtain optimal MAPF solution by checking satisfiability of $\mathcal{F}\left(\xi_{0}\right)$, $\mathcal{F}\left(\xi_{0}+1\right), \mathcal{F}\left(\xi_{0}+2\right), \ldots$ until the first satisfiable $\mathcal{F}(\xi)$ is met $\left(\xi_{0}\right.$ is the lower bound for the sum-of-costs calculated as the sum of lengths of shortest paths). This is possible due to monotonicity of MAPF solvability with respect to increasing values of common cumulative objectives. The framework of SAT-based solving is shown in pseudo-code in Algorithm 2.

The advantage of the SAT-based approach is that state-ofthe-art SAT solvers can be used for determining satisfiability of $\mathcal{F}(\xi)$ [Audemard and Simon, 2009].

\section{Details of MDD-SAT Encoding}

Construction of $\mathcal{F}(\xi)$ as used in MDD-SAT relies on the time expansion of underlying graph $G$. Having $\xi$, the basic variant of time expansion determines the maximum number of time steps $\mu$ (makespan) such that every possible solution of with the sum-of-costs less than or equal to $\xi$ fits in $\mu$ timesteps.

The time expansion makes copies of vertices $V$ for each timestep $t=0,1,2, \ldots, \mu$. That is, we have vertices $v^{t}$ for each $v \in V$ and time step $t$. Edges from $G$ are converted to directed edges interconnecting timesteps in the time expansion. Directed edges $\left(u^{t}, v^{t+1}\right)$ are introduced for $t=1,2, \ldots, \mu-1$ whenever there is $\{u, v\} \in E$. Wait actions are modeled by introducing edges $\left(u^{t}, u^{t+1}\right)$. A directed path in the time expansion corresponds to trajectory of an agent in time. Hence the modeling task now consists in construction of a formula in which satisfying assignments correspond to directed paths from $\alpha_{0}^{0}\left(a_{i}\right)$ to $\alpha_{+}^{\mu}\left(a_{i}\right)$ in the time expansion.

Assume that we have time expansion $T E G_{i}=\left(V_{i}, E_{i}\right)$ for agent $a_{i}$. Propositional variable $\mathcal{X}_{v}^{t}\left(a_{j}\right)$ is introduced for every vertex $v^{t}$ in $V_{i}$. The semantics of $\mathcal{X}_{v}^{t}\left(a_{i}\right)$ is that it is TRUE if and only if agent $a_{i}$ resides in $v$ at time step $t$. Similarly we introduce $\mathcal{E}_{u, v}^{t}\left(a_{i}\right)$ for every directed edge $\left(u^{t}, v^{t+1}\right)$ in $E_{i}$. Analogously the meaning of $\mathcal{E}_{u, v}^{t}\left(a_{i}\right)$ is: it is TRUE if 
and only if agent $a_{i}$ traverses edge $\{u, v\}$ between time steps $t$ and $t+1$.

Finally constraints are added so that truth assignment are restricted to those that correspond to valid solutions of a given MAPF. Added constraints together ensure that $\mathcal{F}(\xi)$ is a complete propositional model for given MAPF.

We here illustrate the model by showing few representative constraints. We omit here constraints that concern objective function. For the detailed list of constraints we refer the reader to [Surynek et al., 2016].

We already know that collisions among agents are eliminated by the $O C C_{\leq 1}$ constraint. $O C C_{\leq 1}$ can be expressed on top of $\mathcal{X}_{v}^{t}\left(a_{i}\right)$ variables by the following constraint for every $v \in V$ and timestep $t$ :

$$
\sum_{a_{i} \in A \mid v^{t} \in V_{i}} \mathcal{X}_{v}^{t}\left(a_{i}\right) \leq 1
$$

There are various ways how to translate the constraint using propositional clauses. One efficient way is to introduce $\neg \mathcal{X}_{v}^{t}\left(a_{i}\right) \vee \neg \mathcal{X}_{v}^{t}\left(a_{j}\right)$ for all possible pairs of $a_{i}$ and $a_{j}$.

Next, there is a constraint stating that if agent $a_{i}$ appears in vertex $u$ at time step $t$ then it has to leave through exactly one edge $\left(u^{t}, v^{t+1}\right)$ :

$$
\begin{array}{r}
\mathcal{X}_{u}^{t}\left(a_{i}\right) \Rightarrow \bigvee_{\left(u^{t}, v^{t+1}\right) \in E_{i}} \mathcal{E}_{u, v}^{t}\left(a_{i}\right) \\
\sum_{v^{t+1} \mid\left(u^{t}, v^{t+1}\right) \in E_{i}} \mathcal{E}_{u, v}^{t}\left(a_{i}\right) \leq 1
\end{array}
$$

Similarly, the target vertex of any movement except wait action must be empty. This is ensured by the following constraint for every $\left(u^{t}, v^{t+1}\right) \in E_{i}$ :

$$
\mathcal{E}_{u, v}^{t}\left(a_{i}\right) \Rightarrow \bigwedge_{a_{j} \in A \mid a_{j} \neq a_{i} \wedge v^{t} \in V_{j}} \neg \mathcal{X}_{v}^{t}\left(a_{j}\right)
$$

Other constraints ensure that truth assignments to variables per individual agents form paths. That is if agent $a_{i}$ enters an edge it must leave the edge at the next time step.

$$
\mathcal{E}_{u, v}^{t}\left(a_{i}\right) \Rightarrow \mathcal{X}_{v}^{t}\left(a_{i}\right) \wedge \mathcal{X}_{v}^{t+1}\left(a_{i}\right)
$$

A common measure how to reduce the number of decision variables derived from the time expansion is the use of multivalue decision diagrams (MDDs) [Sharon et al., 2013]. The basic observation that holds for MAPF is that an agent can reach vertices in the distance $d$ (distance of a vertex is measured as the length of the shortest path) from the current position of the agent no earlier than in the $d$-th time step. Analogous observation can be made with respect to the distance from the goal position.

Above observations can be utilized when making the time expansion of $G$. For a given agent, we do not need to consider all vertices at time step $t$ but only those that are reachable in $t$ timesteps from the initial position and that ensure that the goal can be reached in the remaining $\mu-t$ timesteps.

The combination of SAT-based approach and MDD time expansion led to the MDD-SAT algorithm described in [Surynek et al., 2016] that currently represents state-of-theart in SAT-based MAPF solving.

\section{Combining SMT and CBS}

A natural relaxation from the complete propositional model is an incomplete propositional model where instead of the equivalence between solving MAPF and the formula we require an implication only.

Definition 4 (incomplete propositional model). Propositional formula $\mathcal{H}(\xi)$ is an incomplete propositional model of $M A P F \Sigma$ if the following condition holds:

$$
\mathcal{H}(\xi) \text { is satisfiable } \Leftarrow \Sigma \text { has a solution of sum-of-costs } \xi \text {. }
$$

A close look at CBS reveals that it operates similarly as problem solving in satisfiability modulo theories (SMT) [Bofill et al., 2012]. SMT divides satisfiability problem in some complex theory $T$ into an abstract propositional part that keeps the Boolean structure of the decision problem and a simplified decision procedure $D E C I D E_{T}$ that decides fragment of $T$ restricted on conjunctive formulae. A general $T$ formula $\Gamma$ is transformed to a propositional skeleton by replacing atoms with propositional variables. The SAT solver then decides what variables should be assigned TRUE in order to satisfy the skeleton - these variables tells what atoms hold in $\Gamma . D E C I D E_{T}$ then checks if the conjunction of atoms assigned TRUE is valid with respect to axioms of $T$. If so then satisfying assignment is returned. Otherwise a conflict from $D E C I D E_{T}$ (often called a lemma) is reported back and the skeleton is extended with a constraint forbidding the conflict. This is the basic SMT solving process; more advanced schemes exist where the SAT solver and $T$ are integrated more tightly [Nieuwenhuis et al., 2006].

The above observation led us to the idea to rephrase CBS in terms of SMT. The abstract propositional part working with the skeleton will be taken from MDD-SAT provided that only constraints ensuring that assignments form valid paths interconnecting starting positions with goals will be preserved. Other constraints for collision avoidance will be omitted initially. This will result in an incomplete propositional model.

The paths validation procedure will act as $D E C I D E_{T}$ and will report back the set of conflicts found in the current solution. Hence axioms of $T$ will be represented by the movement rules of MAPF. We call the resulting algorithm SMT-CBS and it is shown in pseudo-code as Algorithm 3. The algorithm simulates the aforementioned SMT solving scheme.

The algorithm is divided into two procedures: SMT-CBS representing the main loop and SMT-CBS-Fixed solving the input MAPF for fixed cost $\xi$. The major difference from the standard CBS is that there is no branching at the highlevel. The high-level SMT-CBS roughly correspond to the main loop of MDD-SAT. The set of conflicts is iteratively collected during the entire execution of the algorithm. Procedure encode from MDD-SAT is replaced with encode-Basic that produces encoding that ignores specific movement rules (collisions between agents) but in contrast to encode it encodes collected conflicts into $\mathcal{H}(\xi)$.

The conflict resolution in the standard CBS implemented as high-level branching is here represented by refinement of $\mathcal{H}(\xi)$ with disjunction (line 20). The presented SMT-CBS can eventually build the same formula as MDD-SAT but this is done lazily in SMT-CBS. 


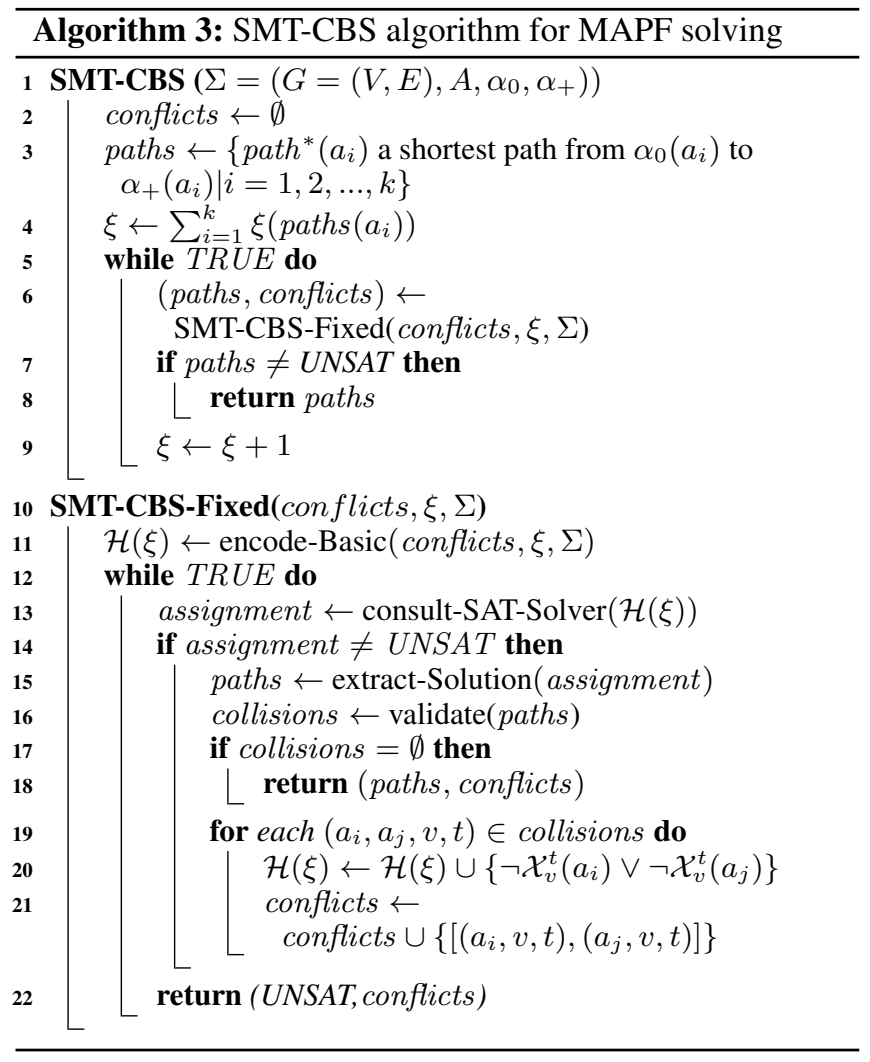

In the line with Observations 1 and 2 such approach may save resources as a solution may be found or its existence can be ruled out prior to adding all constraints.

\section{Experiments}

We performed experiments with the new SMT-CBS algorithm on standard benchmarks [Boyarski et al., 2015; Sharon et al., 2013]. Representative part of results is presented.

\subsection{Benchmarks and Setup}

We implemented SMT-CBS in C++ on top of the Glucose 4 SAT solver [Audemard and Simon, 2009] that ranks among the best SAT solvers according to recent SAT solver competitions [Balyo et al., 2017]. Whenever possible the SAT solver is consulted in the incremental mode. The standard CBS has been re-implemented in $\mathrm{C}++$ from scratch since the original implementation written in Java does support only grids but not general graphs [Sharon et al., 2015] that we need in our tests. We also compared SMT-CBS to one of the most recent $\mathrm{C}++$ versions of ICBS [Boyarski et al., 2015] that implements rectangle reasoning [Li et al., 2019]. And finally we took existing implementation of MDD-SAT also written in $\mathrm{C}++$.

In CBS we implemented preference of resolving cardinal conflicts [Boyarski et al., 2015]. Without this heuristic, CBS exhibited poor performance. In SMT-CBS we initially tried to resolve against single cardinal conflict too but eventually it turned out to be more efficient to resolve against all discovered conflicts (the presented pseudo-code shows this variant).
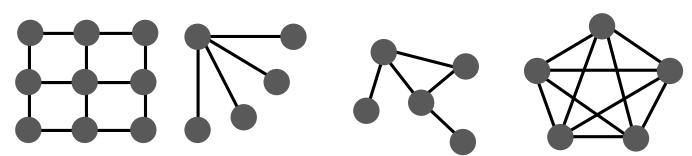

Figure 2: Examples of grid, star, random graph, and clique.

All experiments were run on a Ryzen 7 CPU 3.0 Ghz under Kubuntu linux 16 with 16 GB RAM. ${ }^{2}$.

We divided the experimental evaluation into two categories of tests. The first part of experimental evaluation has been done on diverse instances consisting of small graphs: 4connected open grid of size $8 \times 8$, random graphs containing $20 \%$ of random edges, star graphs, and cliques (see Figure 2). Cliques, random graphs, and stars consisted of 16 vertices.

The initial and goal configurations of agents have been generated randomly in all tests.

The second part of experimental evaluation took place on large 4-connected maps taken from Dragon Age [Sharon et al., 2015; Sturtevant, 2012]. In contrast to small instances, these were only sparsely populated with agents. Initial and goal configuration were generated at random again.

We varied the number of agents in MAPF instances to obtain instances of various difficulties. For each number of agents we generated 10 random instances.

The timeout was set to 1000 seconds. Presented results were obtained from instances finished under this timeout.

\subsection{Comparison on Small Graphs}

Tests on small graphs were focused on the runtime comparison. Results are shown Figure 3 - sorted runtimes are presented, hence easy instances are to the left while hard instances are to the right.

CBS performs well in easy instances but its performance degrades quickly. Both MDD-SAT and SMT-CBS are faster for instances containing more agents. For hardest instances solvable under the timeout (the hardest $8 \times 8$ instance contains 20 agents) the performance of MDD-SAT and SMTCBS is roughly the same. The most interesting situation can be observed in the middle with instances of medium difficulty; here SMT-CBS dominates over MDD-SAT by factor of 2 to 10 .

These observations are in the line with our hypotheses. When agents interacts too much, SMT-CBS produces the same formula as MDD-SAT does, that is why we see similar performance for hardest instances. With less interacting agents SMT-CBS does not need to deal with all potential conflicts and is faster than MDD-SAT.

\subsection{Evaluation on Large Maps}

The second category of tests was focused on the performance of CBS, MDD-SAT and SMT-CBS on large maps. In the three structurally different maps up to 50 agents were placed randomly. Again we had 10 random instances per each number of agents.

\footnotetext{
${ }^{2}$ To enable reproducibility of presented results we provide the complete source code and experimental data: http://users.fit.cvut.cz/ surynpav/research/ijcai2019
} 

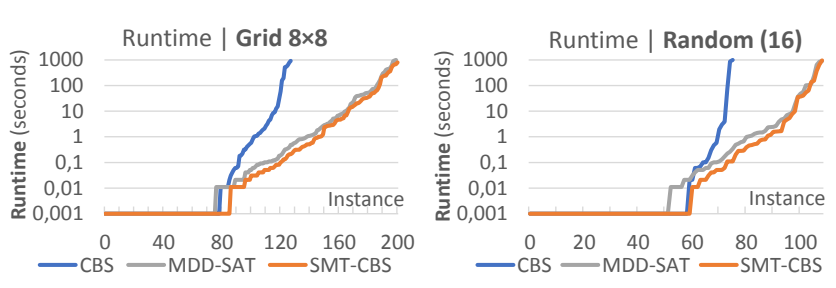

Runtime | Clique (16)
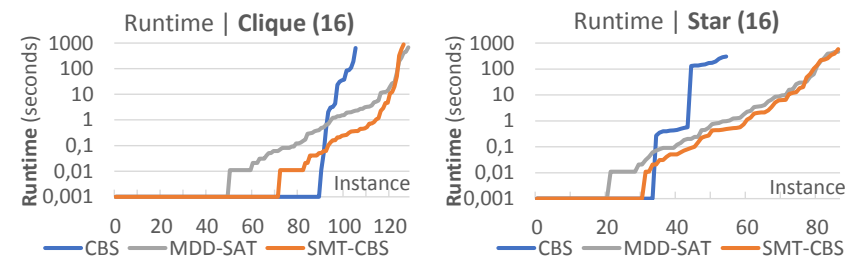

Figure 3: Runtime comparison of CBS, MDD-SAT and SMT-CBS on various small graphs.

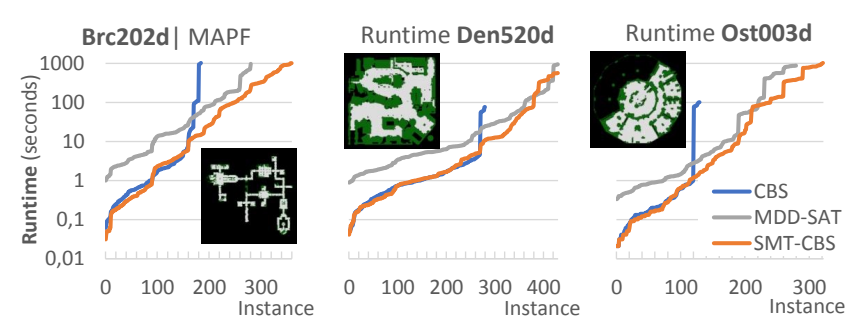

Figure 4: Runtime comparison of CBS, MDD-SAT and SMT-CBS on large maps.

Sorted runtimes are shown in Figure 4. There is no significant difference between CBS and SMT-CBS in easier cases but MDD-SAT lags behind. The situation changes after going into the medium difficulty region where runtimes of CBS go quickly up while SMT-CBS maintains significant advantage over MDD-SAT (factor 2 to 5). The performance of SMTCBS and MDD-SAT eventually meets in the hard region.

In addition to runtime comparison, we compared the number of clauses generated by MDD-SAT and SMT-CBS. This comparison is focused on verifying if SMT-CBS terminates prior to adding all possible constraints. Sorted numbers of clauses are shown in Figure 5 from which we can clearly see that SMT-CBS generates order of magnitudes fewer clauses than MDD-SAT. This is directly reflected in smaller memory consumption by SMT-CBS.
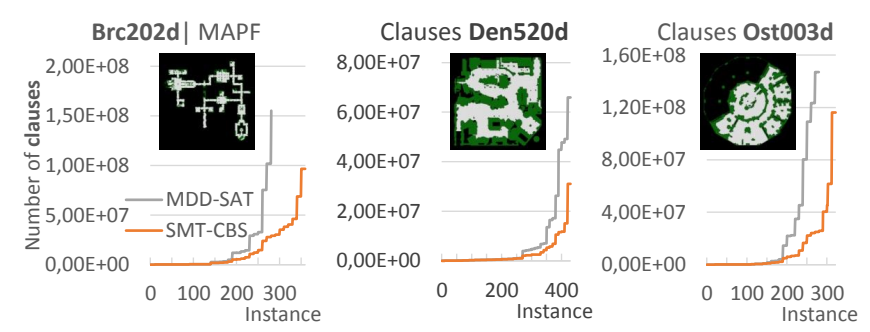

Figure 5: Clauses generated by MDD-SAT and SMT-CBS.

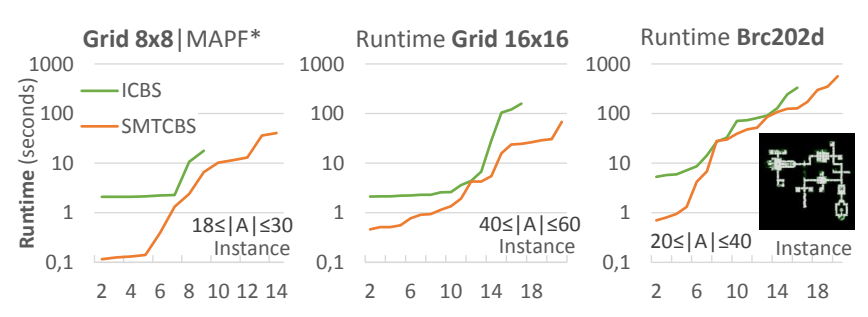

Figure 6: Comparison of SMT-CBS to ICBS implementing rectangular reasoning.

\subsection{SMT-CBS and Recent Progress in ICBS}

Comparing our implementaiton of SMT-CBS with the recent version of ICBS featuring various heuristics such as rectangular reasoning indicated that SMT-CBS has a significant advantage on small grids ${ }^{3}$ (sorted runtimes presented in Figure 6). As instances get harder, runtime of ICBS goes quickly up reaching the timeout of 1000 seconds earlier than SMTCBS. In large maps, SMT-CBS and ICBS are closer to each other however SMT-CBS was still faster for a larger set of instances.

In this experiment we used a version of MAPF (denoted

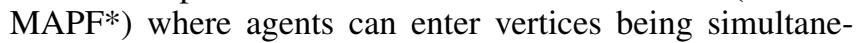
ously vacated by other agents. This induced necessity to introduce edge conflicts in SMT-CBS.

The important observation is that SMT-CBS and ICBS differ on individual instances greatly. It often happens that an instance easy for ICBS is hard for SMT-CBS and vice versa.

\section{Conclusion}

We suggested a new MAPF solving method called SMTCBS that combines advantages of MDD-SAT - fast solving in highly constrained cases, and advantages of CBS - fast solving in large sparse environments.

The new algorithm outperforms CBS and ICBS and improves the performance of MDD-SAT in terms of the size of generated formulae as well as in runtime. Experiments confirmed our hypotheses that SMT-CBS is able to produce solution well before all constraints are added to the encoding which altogether leads to faster solving.

The performance gap between MDD-SAT and SMT-CBS is not as big as the gap in the number of generated clauses. The explanation of this is that SMT-CBS needs to call the SAT solver more times than MDD-SAT does which represents an extra overhead.

For the future work we plan to further generalize SMTCBS approach; geometric agents in a continuous environment represent one promising direction.

\section{Acknowledgments}

This work has been supported by GAČR - the Czech Science Foundation, grant registration number 19-17966S.

\footnotetext{
${ }^{3}$ The implementation of ICBS does not support general graphs.
} 


\section{References}

[Audemard and Simon, 2009] G. Audemard and L. Simon. Predicting learnt clauses quality in modern SAT solvers. In IJCAI, pages 399-404, 2009.

[Balyo et al., 2017] Tomás Balyo, Marijn J. H. Heule, and Matti Järvisalo. SAT competition 2016: Recent developments. In $A A A I$, pages 5061-5063, 2017.

[Basile et al., 2012] Francesco Basile, Pasquale Chiacchio, and Jolanda Coppola. A hybrid model of complex automated warehouse systems - part I: modeling and simulation. IEEE Trans. Automation Science and Engineering, 9(4):640-653, 2012.

[Biere et al., 2009] A. Biere, A. Biere, M. Heule, H. van Maaren, and T. Walsh. Handbook of Satisfiability: Volume 185 Frontiers in Artificial Intelligence and Applications. IOS Press, 2009.

[Bofill et al., 2012] Miquel Bofill, Miquel Palahí, Josep Suy, and Mateu Villaret. Solving constraint satisfaction problems with SAT modulo theories. Constraints, 17(3):273303, 2012.

[Boyarski et al., 2015] E. Boyarski, A. Felner, R. Stern, G. Sharon, D. Tolpin, O. Betzalel, and S. Shimony. ICBS: improved conflict-based search algorithm for multi-agent pathfinding. In IJCAI, pages 740-746, 2015.

[de Wilde et al., 2014] B. de Wilde, A. ter Mors, and C. Witteveen. Push and rotate: a complete multi-agent pathfinding algorithm. JAIR, 51:443-492, 2014.

[Dresner and Stone, 2008] K. Dresner and P. Stone. A multiagent approach to autonomous intersection management. JAIR, 31:591-656, 2008.

[Felner et al., 2018] Ariel Felner, Jiaoyang Li, Eli Boyarski, Hang Ma, Liron Cohen, T. K. Satish Kumar, and Sven Koenig. Adding heuristics to conflict-based search for multi-agent path finding. In Proceedings of ICAPS 2018, pages 83-87, 2018.

[Kautz and Selman, 1999] Henry A. Kautz and Bart Selman. Unifying sat-based and graph-based planning. In Proceedings of the Sixteenth International Joint Conference on Artificial Intelligence, IJCAI 1999, pages 318-325, 1999.

[Kim et al., 2014] D.-G Kim, Katsutoshi Hirayama, and G.$\mathrm{K}$ Park. Collision avoidance in multiple-ship situations by distributed local search. Journal of Advanced Computational Intelligence and Intelligent Informatics, 18:839848, 092014.

[Kornhauser et al., 1984] Daniel Kornhauser, Gary L. Miller, and Paul G. Spirakis. Coordinating pebble motion on graphs, the diameter of permutation groups, and applications. In FOCS, 1984, pages 241-250, 1984.

[Li et al., 2019] Jiaoyang Li, Daniel Harabor, Peter Stuckey, Hang Ma, and Sven Koenig. Symmetry-breaking constraints for grid-based multi-agent path finding. In $A A A I$. AAAI Press, 2019.

[Luna and Bekris, 2011] R. Luna and K. E. Bekris. Push and swap: Fast cooperative path-finding with completeness guarantees. In IJCAI, pages 294-300, 2011.
[Miltzow et al., 2016] Tillmann Miltzow, Lothar Narins, Yoshio Okamoto, Günter Rote, Antonis Thomas, and Takeaki Uno. Approximation and hardness of token swapping. In ESA 2016, volume 57 of LIPICs, pages 66:166:15. Schloss Dagstuhl, 2016.

[Nieuwenhuis et al., 2006] Robert Nieuwenhuis, Albert Oliveras, and Cesare Tinelli. Solving SAT and SAT modulo theories: From an abstract davis-putnamlogemann-loveland procedure to $\operatorname{dpll}(T)$. J. ACM, 53(6):937-977, 2006.

[Ratner and Warmuth, 1986] Daniel Ratner and Manfred K. Warmuth. Finding a shortest solution for the $\mathrm{N} x \mathrm{~N}$ extension of the 15-puzzle is intractable. In $A A A I$, pages 168 $172,1986$.

[Ryan, 2008] Malcolm R. K. Ryan. Exploiting subgraph structure in multi-robot path planning. J. Artif. Intell. Res. (JAIR), 31:497-542, 2008.

[Sharon et al., 2013] G. Sharon, R. Stern, M. Goldenberg, and A. Felner. The increasing cost tree search for optimal multi-agent pathfinding. Artif. Intell., 195:470-495, 2013.

[Sharon et al., 2015] G. Sharon, R. Stern, A. Felner, and N. Sturtevant. Conflict-based search for optimal multiagent pathfinding. Artif. Intell., 219:40-66, 2015.

[Silver, 2005] D. Silver. Cooperative pathfinding. In AIIDE, pages 117-122, 2005.

[Standley, 2010] T. Standley. Finding optimal solutions to cooperative pathfinding problems. In $A A A I$, pages 173 $178,2010$.

[Sturtevant, 2012] Nathan R. Sturtevant. Benchmarks for grid-based pathfinding. Computational Intelligence and AI in Games, 4(2):144-148, 2012.

[Surynek et al., 2016] Pavel Surynek, Ariel Felner, Roni Stern, and Eli Boyarski. Efficient SAT approach to multiagent path finding under the sum of costs objective. In ECAI, pages 810-818, 2016.

[Surynek, 2010] Pavel Surynek. An optimization variant of multi-robot path planning is intractable. In AAAI 2010. AAAI Press, 2010.

[Surynek, 2017] Pavel Surynek. Time-expanded graphbased propositional encodings for makespan-optimal solving of cooperative path finding problems. Ann. Math. Artif. Intell., 81(3-4):329-375, 2017.

[Wilson, 1974] Richard M Wilson. Graph puzzles, homotopy, and the alternating group. Journal of Combinatorial Theory, Series B, 16(1):86 - 96, 1974.

[Yu and LaValle, 2016] Jingjin Yu and Steven M. LaValle. Optimal multirobot path planning on graphs: Complete algorithms and effective heuristics. IEEE Trans. Robotics, 32(5):1163-1177, 2016.

[Zhou and Schwager, 2015] Dingjiang Zhou and Mac Schwager. Virtual rigid bodies for coordinated agile maneuvering of teams of micro aerial vehicles. In ICRA 2015, pages 1737-1742, 2015. 